\title{
APROXIMACIÓN SISTÉMICA DE LA SOSTENIBILIDAD EN LA PRODUCCIÓN DE BIOETANOL
}

\author{
SYSTEMIC APPROACH TO SUSTAINABILITY \\ IN THE BIOETHANOL PRODUCTION
}

\author{
Danny Ibarra Vega ${ }^{1}$ \\ Gerard Olivar Tost ${ }^{2}$ \\ ${ }^{1}$ Doctorando en Ingeniería, Universidad Nacional de Colombia. Docente e investigador \\ del Programa de Ingeniería Ambiental de la Universidad Sergio Arboleda. \\ ${ }^{2}$ Doctor en Ciencias - Matemáticas por la Universitat Politécnica de Catalunya, \\ Barcelona, España. Profesor titular Universidad Nacional de Colombia. \\ 1 ingdanny09@hotmail.com \\ 2 golivar@unal.edu.co
}

\section{Resumen}

En este artículo se presenta la descripción del problema de sostenibilidad que enfrentan los sistemas de producción de biocombustibles, en específico la producción de bioetanol en Colombia, desde una preceptiva sistémica desarrollada por medio de diagramas causales del problema. Para comprender la sostenibilidad de la bioenergía y los biocombustibles, existe un conjunto de indicadores de sostenibilidad que pueden ser útiles para las economías que están incursionando en la bioenergía o que tienen una producción consolidada, como es el caso colombiano. De esta forma, se describen las etapas de producción en donde estos indicadores aplican para el contexto colombiano en la producción de bioetanol de caña de azúcar. Finalmente, para ilustrar como se deben interconectar los indicadores en el proceso productivo, se obtuvo un diagrama causal que emerge de la conceptualización sistémica del problema éste permitió la captura cualitativa de los principales atributos del sistema, con sus respectivas relaciones causales y estructuras de realimentación típicos del pensamiento sistémico.

Palabras clave: biocombustibles, diagrama causal, indicadores, sostenibilidad, pensamiento sistémico.

\begin{abstract}
This paper presents a description of the sustainability problem faced by biofuel production systems, specifically the production of bioethanol in Colombia, from a systemic precept developed by causal diagrams of the problem. To understand the sustainability of bioenergy and biofuels, there is a set of 24
\end{abstract}


sustainability indicators that may be useful for economies that are entering bioenergy or have a consolidated production such as the Colombian case. In this way, the production stages are described where these indicators apply to the Colombian context of sugarcane production. Finally, to illustrate how the indicators are interconnected in the production process, a causal diagram was obtained that emerges from the systemic conceptualization of the problem, which allowed the qualitative capture of the main attributes of the system with the respective causal relationships and typical feedback structures of the system thinking.

Keywords: biofuels, causal diagram, indicators, sustainability, system thinking.

\section{Introducción}

Los biocombustibles han sido denominados como una opción para disminuir la dependencia de los combustibles fósiles, así como una alternativa de mitigación de las emisiones de gases de efecto invernadero, fuente para la diversificación de la canasta energética, y una oportunidad para la creación de trabajo y desarrollo rural (Scarlat \& Dallemand, 2011; Espinoza et al., 2017). Los biocombustibles más conocidos a nivel mundial son el bioetanol y el biodiesel. El bioetanol es un tipo de biocombustible que puede ser utilizado de manera directa o como aditivo oxigenante de la gasolina (Sánchez \& Cardona, 2008). Este, se obtiene a partir de materias primas ricas en azucares fermentables como la caña de azúcar, el maíz y la remolacha azucarera. La producción de bioetanol y biodiesel se encuentra posicionada en Colombia y se producen a escala industrial desde el año 2005. Sin embargo, hay algunas preocupaciones sobre los posibles impactos directos e indirectos con respecto al cambio climático, la seguridad alimentaria, afectaciones ambientales y el desarrollo económico que aún son discutidas en distintos contextos (Valencia \& Cardona 2014; Esteves, 2013).

En este artículo, se aborda de manera general la descripción de la sostenibilidad de la producción de biocombustibles, desde una perspectiva sistémica, por medio de la vinculación indicadores de sostenibilidad de la Global Bioenergy Parnership (GBEP, 2011), (En Inglés) a la cadena de suministro de bioetanol de caña de azúcar. Para esto, se partió de la construcción un diagrama causal que representa una cadena productiva de bioetanol de caña de azúcar en Colombia, posteriormente se vincularon los indicadores al diagrama causal y se describe la forma en que deberían integrarse y evaluarse estos indicadores.

\section{Metodología}

Para el desarrollo de este artículo se recopiló la información relacionada sobre la producción de biocombustibles, específicamente bioetanol de caña de azúcar en el contexto colombiano. A partir de allí, se construyó una cadena de suministro teórica que se aproxima a la realidad, esta construcción se realizó con los fundamentos conceptuales de la metodología Dinámica de Sistemas, la cual permite construir modelos mentales donde se concibe cualquier aspecto del mundo como la interacción causal entre atributos que lo describen (Ibarra \& Redondo, 2015). Así, se construyó la representación sistémica con flechas y variables, denominada 'diagrama causal'. Una vez obtenido el diagrama causal de la cadena de suministro, se identificaron de manera cualitativa los indicadores de sostenibilidad, para este trabajo se muestran seis indicadores 
seleccionados de un estudio previo (Ibarra, 2017) que aplican para una cadena de producción de bioetanol de caña en Colombia. Posteriormente se construyó un primer diagrama causal de sostenibilidad donde muestra como ejemplo la forma en que se relacionan los indicadores de sostenibilidad con una cadena productiva de bioetanol.

\section{Contextualización biocombustibles}

Colombia posee las características geográficas y climáticas necesarias para la producción de biocombustibles, las vastas extensiones de tierra disponibles para cultivar materias primas, la mano de obra a precios razonables y las condiciones agroclimáticas adecuadas para el desarrollo de una amplia variedad de cultivos utilizados como materias primas hacen que Colombia se convierta en uno de los países con potencial para la producción de biocombustibles (Flórez, 2011).

El bioetanol en Colombia se produce a partir de caña de azúcar, debido a que la producción de caña de azúcar se encuentra consolidada desde hace años en el país, este tipo de industria pasó de ser solo azucarera para convertirse en clústers de alcoquímica. La caña de azúcar presenta mayor eficiencia energética $(8,3$ $\mathrm{KJ})$ frente a otras materias primas a partir de las cuales se produce etanol, como lo muestra la (Figura 1).

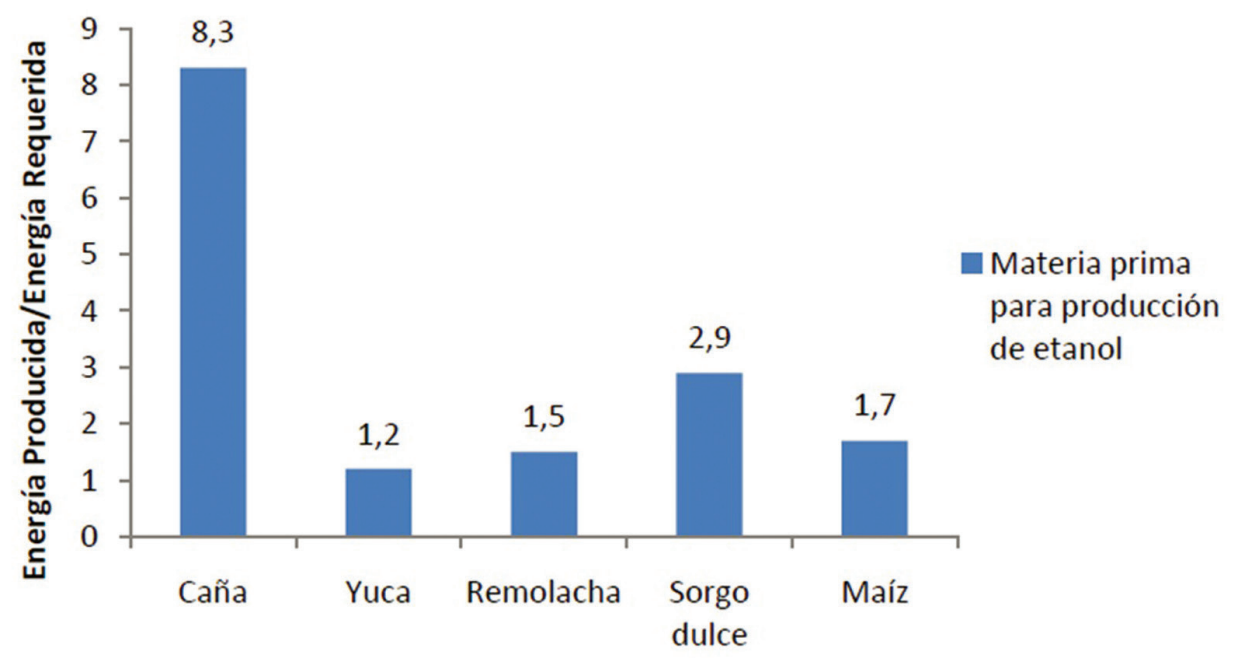

Figura 1. Eficiencia energética Materias primas.

Fuente: MME con base en Goldman Sachs y LMC en el documento Conpes 3510

De acuerdo con Fedesarrollo (2012) y Fedebiocombustibles (2016) la producción de etanol en Colombia se lleva a cabo principalmente en el Valle del río Cauca, principalmente en los departamentos Cauca, Valle del Cauca, Risaralda y Caldas, abarcando 47 municipios. En esta región hay 230.311 hectáreas sembradas en caña para azúcar, de las cuales el $24 \%$ corresponde a tierras propias de los ingenios y
$76 \%$ el restante a más de 2.000 cultivadores de caña. Dichos cultivadores abastecen a los 13 ingenios de la región (La Cabaña, Carmelita, Manuelita, María Luisa, Mayagüez, Pichichí, Risaralda, San Carlos, Tumaco, Ríopaila-Castilla, Incauca y Providencia). La producción de bioetanol en Colombia pasó de ser 25 millones de litros en 2005 a 456 millones de litros en 2015, como lo muestra la (Figura 2). 


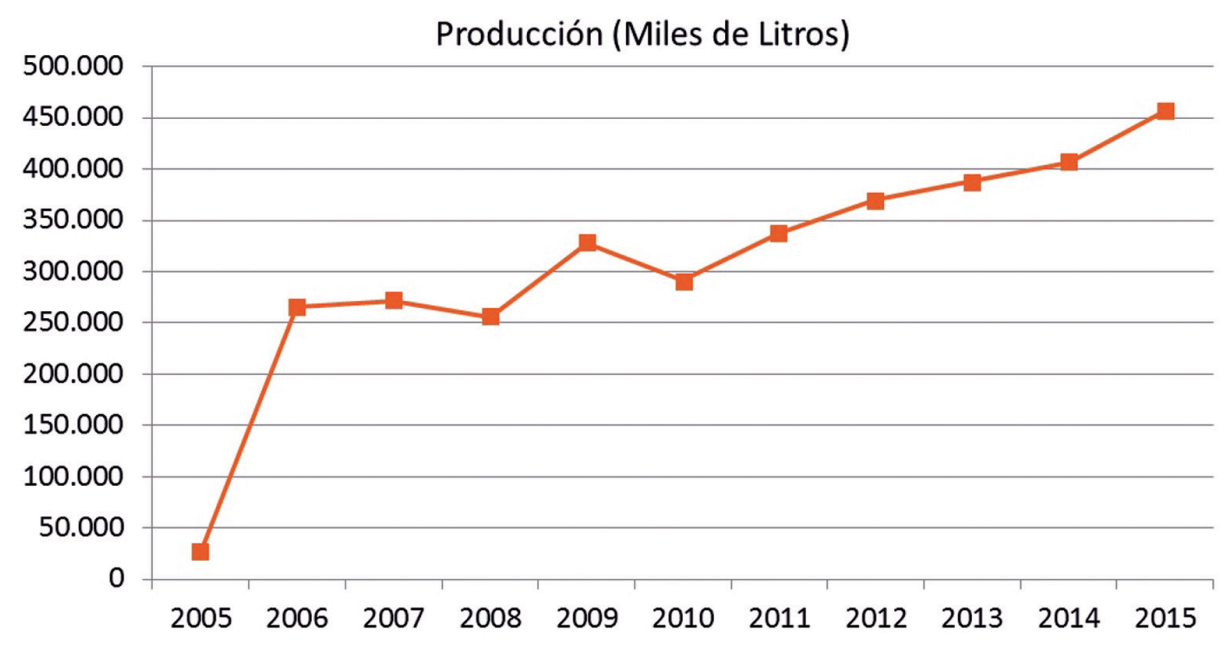

Figura 2. Producción de alcohol carburante.

Modificado de: (Asocaña - Balance azucarero 2012 y 2016)

\section{La producción de Bioetanol de Caña}

El bioetanol $\left(\mathrm{C}_{2} \mathrm{H}_{5} \mathrm{OH}\right)$ de primera generación se produce de manera tradicional por un proceso que consiste de tres etapas principalmente:
Fermentación, Destilación, y Deshidratación. Teniendo en cuenta las diferentes materias primas que se puedan emplear como lo muestra la (Tabla 1).

Tabla 1. Fuentes y cultivos para bioetanol.

\begin{tabular}{|c|c|c|c|c|c|}
\hline $\begin{array}{l}\text { Fuente de } \\
\text { Carbono }\end{array}$ & $\begin{array}{l}\text { Cultivo } \\
\text { agrícola }\end{array}$ & $\begin{array}{c}\text { Rendimiento } \\
\text { (Litros/Tonelada } \\
\text { de cosechado) }\end{array}$ & $\begin{array}{c}\text { Rendimiento } \\
\text { (Litros/Hectárea) }\end{array}$ & $\begin{array}{c}\text { Costo de } \\
\text { Producción } \\
\text { (USD/Litro) }\end{array}$ & País \\
\hline \multirow{5}{*}{ Sacarosa } & Remolacha (Jugo) & 100 & 7000 & 0,48 & Unión Europea \\
\hline & Caña (Jugo) & $70-85$ & 6000 & 0,21 & Brasil \\
\hline & \multirow{2}{*}{ Caña (Melaza) } & 10 & 590 & 0,32 & India \\
\hline & & 10 & 730 & $0,23-0,37$ & México \\
\hline & Sorgo & $56-90$ & $2500-4000$ & & Suecia \\
\hline \multirow{3}{*}{ Almidón } & Maíz & 400 & 3000 & $0,29-0,37$ & Estados Unidos \\
\hline & Trigo & 340 & 2700 & 0,62 & Unión Europea \\
\hline & Maíz/Trigo paja & 285 Litros/ton paja & & 0,59 & Estados Unidos \\
\hline Celulosa & Bagazo de caña & 55 & 3850 & 0,8 & Chile \\
\hline
\end{tabular}

Fuente: (Chauvet y González, 2008). 
La etapa de fermentación consiste en convertir los azúcares presentes en la materia prima (en este caso caña de azúcar) que vienen de la fábrica, en etanol y gas carbónico, por medio de la acción de levaduras, que realizan esta transformación gracias a su metabolismo natural.

La fermentación se desarrolla por medio de un proceso continuo en reactores donde se llevan a cabo las reacciones químicas de transformación de azúcar en etanol y gas carbónico. A la salida de estos reactores, se obtiene un producto conocido como mosto o vino fermentado que contiene una concentración de alcohol. Además, contiene agua, sólidos y levadura. El mosto o vino fermentado se envía a la siguiente etapa que es la destilación, para continuar de esta manera con la separación del etanol producido (Chauvet \& González, 2008).

En la destilación se purifica el mosto fermentado para obtener alcohol con una mayor pureza que podría llegar a ser del 96\% (v/v). La destilación es un proceso de separación por diferencias en los puntos de ebullición de los componentes de una mezcla, que al ser sometidos al calor, los compuestos más volátiles como el alcohol se evaporan y se concentran en fase de vapor (CUE, 2012).

El mosto fermentado que proviene de la sección de fermentación contiene $9 \%$ de alcohol, además de agua, gases, sólidos disueltos y otros compuestos que deben retirarse. Para ello, se envía a una columna mostera donde se obtienen dos productos: por la parte superior se obtienen gases con una concentración de alcohol entre el $40-50 \%$ v/v que se envían a la segunda columna llamada rectificadora. Por la parte inferior se obtiene una corriente líquida llamada vinaza (CUE, 2012).

Posteriormente viene el proceso de deshidratación, donde el alcohol purificado en la destilación contiene entre un $96 \% \mathrm{v} / \mathrm{v}$ de etanol y $4 \% \mathrm{v} / \mathrm{v}$ de agua. Para que este alcohol pueda ser usado como combustible, es necesario retirarle más agua. La deshidratación tiene lugar en los tamices moleculares, que por medio de una resina sintética retiene el agua contenida en el alcohol rectificado, para obtener como producto alcohol deshidratado con una concentración mínima de $99.5 \%$ de etanol, cumpliendo con las especificaciones para su uso como alcohol carburante. El alcohol deshidratado o alcohol anhidro se condensa y enfría para ser almacenado (Sánchez, 2008; Valencia 2013).

\section{Uso y mezcla del Bioetanol en Colombia}

Las políticas del gobierno colombiano apuntan a que la producción de bioetanol sigan en crecimiento, teniendo en cuenta el alto potencial que tiene el país para esto. De acuerdo con el Decreto 1135 de 2009, del Ministerio de Minas y Energía, a partir del 10 de enero del año 2012 los vehículos automotores hasta 2000 cm3 de cilindrada que se fabriquen, ensamblen, importen, distribuyan y comercialicen en el país y que requieran para su funcionamiento gasolinas, deberán estar acondicionados para que sus motores funcionen con sistema Flexfuel (E85), es decir, que puedan funcionar normalmente utilizando indistintamente gasolinas b'asicas o mezclas compuestas por gasolina básica de origen fósil con al menos $85 \%$ de alcohol carburante. Sin embargo desde el año 2005 la gasolina que se usa en las principales ciudades del país comenzó a denominarse $\mathrm{B} 10$, esto quiere decir un combustible con 10 $\%$ de Bioetanol y $90 \%$ de Gasolina, pese a este mandato del gobierno, hubo la necesidad de reducir el porcentaje a B8 ya que la producción no alcanza para el suministro nacional (Ver Figura 3). Actualmente todos los departamentos del país cuentan con combustible B8, a excepción del Departamento de la Guajira, Norte de Santander y Arauca, quienes cuentan con combustible importado de Venezuela. 


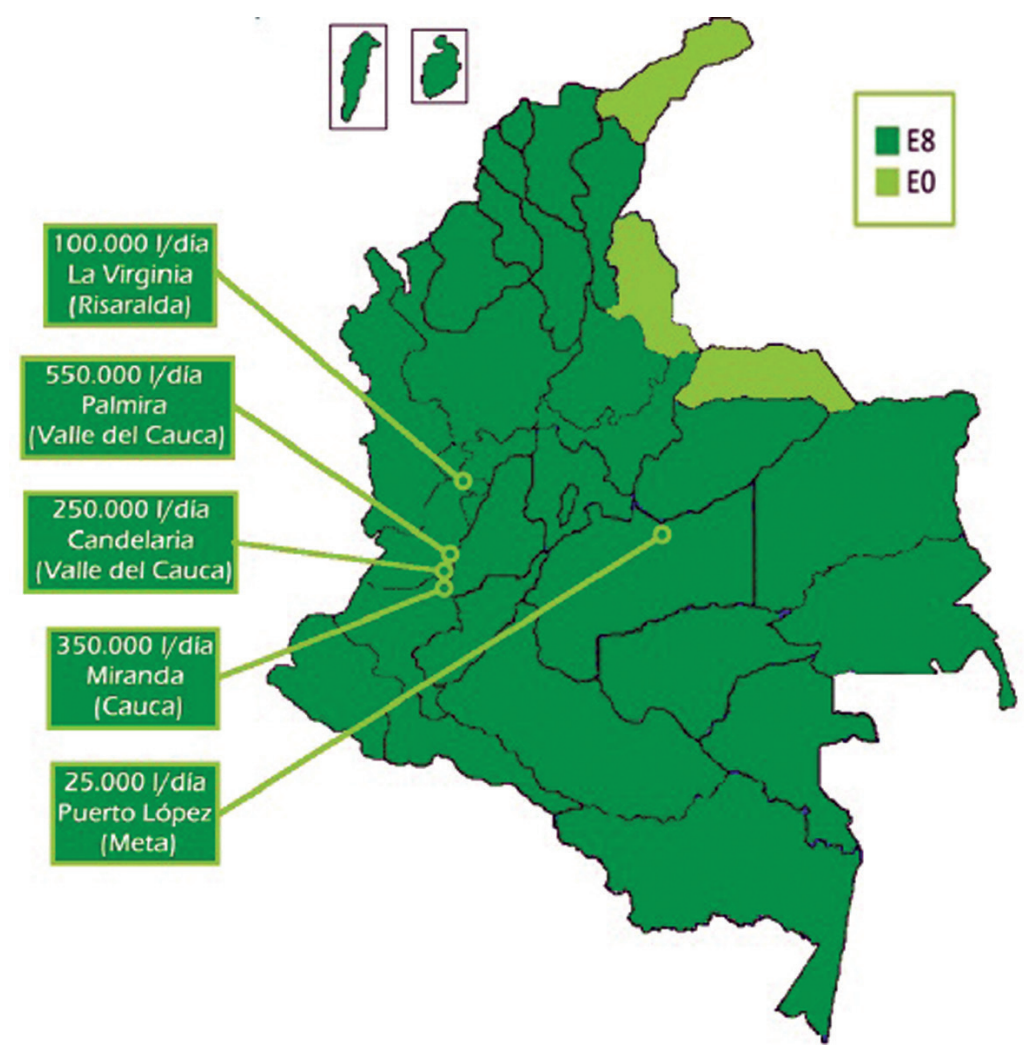

Figura 3. Porcentaje de mezcla de bioetanol en Colombia.

Fuente: FedeBiocombustibles, 2016.

En Colombia, en el año 2015, se produjeron 2,35 millones de tmvc de azúcar a partir de 24,2 millones de toneladas de caña. De alcohol carburante se produjeron 456 millones de litros, destinados a la mezcla con gasolina en una proporción E8 ( $8 \%$ etanol, 92\% gasolina), de acuerdo con el mandato de oxigenación establecido por el gobierno (Asocaña, 2012). De esta manera se refleja el aumento en la producción de litros/año y la capacidad instalada del país para su producción.

Actualmente en Colombia la producción de Bioetanol proviene de la caña de azúcar y debido a la inversión en este sector, la capacidad instalada de producción es de 1'650.000 litros/ día (FedeBiocombustibles, 2016). Se espera que en el 2018 esta capacidad llegue a los 2 millones de litros/día.

En esta misma dirección, se encuentran las políticas del gobierno Colombiano, pues han sido orientadas a que la producción de bioetanol siga en crecimiento, teniendo en cuenta el alto potencial y las vastas extensiones de terreno inexplotado que tiene el país para esto. Cabe resaltar que la apuesta del gobierno es incrementar su producción en el corto y mediano plazo, teniendo en cuenta lineamientos de sostenibilidad que se establecieron en el CONPES 3510 (2008), el cual busca que el país logre una producción eficiente y sostenible, entendiéndose sostenible como un equilibrio entre la dimensión económica, social y ambiental.

Dado lo anterior, es necesario desarrollar herramientas y metodologías que permitan la comprensión de la situación, así como una evaluación que permitan vincular los aspectos e impactos ambientales relacionados con el proceso productivo del bioetanol a lo largo de la cadena de suministro. Es decir, una evaluación sistémica de la sostenibilidad. 
Para esto la FAO a través de la asociación mundial de bioenergía, (GBEP) desarrolló un conjunto 24 indicadores de sostenibilidad, propuestos para la producción de bioenergía, estos basados en los tres pilares del desarrollo sostenible, el pilar ambiental, económico y social. Estos indicadores fueron desarrollados para proporcionar a los formuladores de políticas del sector de bioenergía y a otras partes interesadas, un conjunto de herramientas analíticas que pueden informar sobre el desarrollo de políticas y programas nacionales de bioenergía y así monitorear el impacto de las mismas (GBEP, 2011). Sin embargo, en países en vía de desarrollo como Colombia, aún no es claro cómo se podrían implementar los indicadores de sostenibilidad propuestos, ni cómo se podría evaluar prospectivamente y sistémicamente estos indicadores.

\section{Indicadores de sostenibilidad de biocombustibles}

La Asociación Mundial de Bioenergía (GBEP) ha desarrollado un conjunto veinticuatro indicadores para la evaluación y monitoreo de la sostenibilidad de la bioenergía a niveles nacionales. Los indicadores GBEP pretenden informar a los responsables de formular políticas en los países, sobre los aspectos ambientales, sociales y económicos del sector de la bioenergía en sus países, así como guiarlos hacia políticas que fomenten el desarrollo sostenible. Estos indicadores están basados en los tres pilares de la sostenibilidad; sostenibilidad económica, social y ambiental. Los indicadores del GBEP se enfocan en un nivel nacional y/o regional del mercado, así como en todo el ciclo de vida del biocombustible (Hayashi, Ierland y Zhu, 2014).

Tabla 2. Indicadores de Sostenibilidad del GBEP

\begin{tabular}{|c|c|c|}
\hline Indicadores Ambientales & Indicadores Sociales & Indicadores Económicos \\
\hline $\begin{array}{l}\text { 1. Ciclo de vida de los Gases } \\
\text { de Efecto Invernadero }\end{array}$ & $\begin{array}{l}\text { 9. Asignación y tenencia de la tierra } \\
\text { para nueva producción de bioenergía }\end{array}$ & 17. Productividad \\
\hline 2. Calidad del suelo & $\begin{array}{l}\text { 10. Precio y oferta de una } \\
\text { canasta alimentaria nacional }\end{array}$ & 18. Balance neto de bioenergía \\
\hline $\begin{array}{l}\text { 3. Niveles de cosecha } \\
\text { de recursos madereros }\end{array}$ & 11. Cambio en ingresos & 19. Valor añadido bruto \\
\hline $\begin{array}{l}\text { 4. Emisiones de contaminantes } \\
\text { del aire que no son GEI } \\
\text { (Contaminantes criterio) }\end{array}$ & $\begin{array}{l}\text { 12. Empleos en el sector } \\
\text { de la bioenergía }\end{array}$ & $\begin{array}{l}\text { 20. Cambio en el consumo de } \\
\text { combustibles fósiles y en el } \\
\text { uso tradicional de la biomasa }\end{array}$ \\
\hline 5. Uso y eficiencia del agua & $\begin{array}{l}\text { 13. Cambio en tiempo no pagado } \\
\text { invertido por mujeres y niños } \\
\text { en la recolección de biomasa }\end{array}$ & $\begin{array}{l}\text { 21. Formación y recualificación } \\
\text { de los trabajadores }\end{array}$ \\
\hline 6. Calidad del agua & $\begin{array}{l}\text { 14. Bioenergía usada para ampliar } \\
\text { el acceso a servicios modernos } \\
\text { de energía }\end{array}$ & 22. Diversidad energética \\
\hline $\begin{array}{l}\text { 7. Diversidad biológica } \\
\text { en el paisaje natural }\end{array}$ & $\begin{array}{l}\text { 15. Cambio en la mortalidad y tasas } \\
\text { de enfermedades atribuibles a } \\
\text { humos en espacios cerrados }\end{array}$ & $\begin{array}{l}\text { 23. Infraestructura y logística } \\
\text { para la distribución de } \\
\text { bioenergía }\end{array}$ \\
\hline $\begin{array}{l}\text { 8. Uso de la tierra y cambio de uso } \\
\text { de la tierra relacionados con la } \\
\text { producción de materia prima para } \\
\text { bioenergía }\end{array}$ & $\begin{array}{l}\text { 16. Incidencia de lesiones, } \\
\text { enfermedades y muertes laborales }\end{array}$ & $\begin{array}{l}\text { 24. Capacidad y flexibilidad } \\
\text { del uso de la bioenergía }\end{array}$ \\
\hline
\end{tabular}

Fuente: (GBEP, 2011). 


\section{Resultados \\ Comprensión sistémica}

Dada la relación que existe entre los indicadores de sostenibilidad y una cadena de suministro de etanol en Colombia, se hace entonces necesario incluir una visión sistémica de gestión para poder evaluar la estructura de la cadena de suministro y su relación con los indicadores. Para esto se parte de la teoría de dinámica de sistemas la cual es una metodología para el análisis y resolución de problemas, desarrollada por Jay Forrester y presentada en sus obras (Forrester, 1999a; 1999b). En la cual se construye una hipótesis dinámica que permite la construcción de representaciones sistémicas con flechas y atributos, denominadas diagramas causales, que capturan todas las hipótesis propuestas desde las que se puede aprender del sistema para intervenir sobre él en el ejercicio de decisión (Ibarra \& Redondo, 2015). Dado esto se construyó el diagrama causal, el cual muestra las interrelaciones entre los atributos principales que representan la producción de bioetanol y sus posibles afectaciones ambientales y sociales. Para este trabajo, se muestra un ejemplo de diagrama causal.

\section{Diagrama causal del problema}

A continuación se presentan y definen los principales atributos que fueron identificados para obtener y delimitar el sistema a estudiar, los cuales describen las partes que componen la cadena de suministro de bioetanol.
- Hectáreas de Caña: Es la cantidad de hectáreas de caña sembradas para la producción de bioetanol.

- Incremento Neto: Tasa de incremento hectáreas la caña para la producción.

- Cosechado: Cantidad de Hectáreas que se cosechan y se destinan para la producción de bioetanol.

- Alistamiento de la caña de azúcar: Proceso de limpieza y molienda de la caña cosechada

- Capacidad Instalada: Potencial de producción o volumen máximo de producción bioetanol que tiene el país.

- Jugo de caña: Cantidad de jugo de caña destinado a la fermentación.

- Producción de Bioetanol: Proceso productivo en función de tasa de producción del jugo fermentable y la capacidad instalada

- Bioetanol producido: Acumulación el litros de bioetanol producidos.

- Distribución: Cantidad de bioetanol destinado a la mezcla con gasolina.

- Productividad: Indicador económico que muestra la cantidad de volumen producido por hectárea de caña.

A partir de la identificación de los atributos del sistema, se procede a la construcción del diagrama causal básico de una cadena de suministro genérica, como se presenta a continuación:

Las anteriores hipótesis son representadas como relaciones causales, conformando un diagrama causal (Figura 4). 


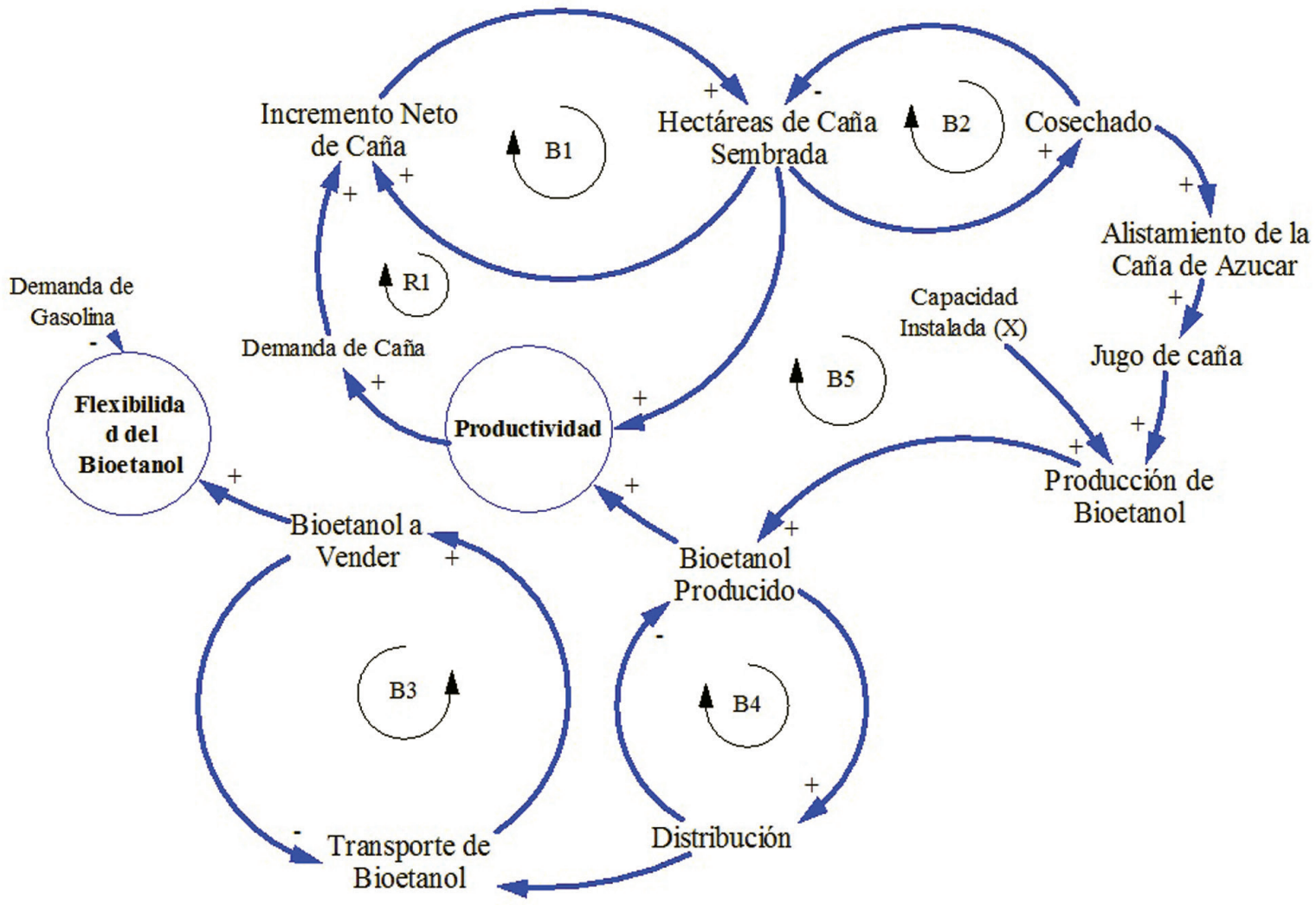

Figura 4. Diagrama causal de la producción de Bioetanol y su relación con los indicadores GBEP. Fuente: Los autores.

Se recuerda que para la lectura del diagrama causal se entiende que si el atributo $x$ aumenta, entonces causa que el atributo $y$ aumente o disminuya, (dependiendo de la polaridad de la relación causal).

Se tienen entonces 6 estructuras o bucles de realimentación, el bucle $\mathrm{B} 1$ y $\mathrm{B} 2$ son la parte de la dinámica propia del cultivo de caña de azúcar, es decir, del incremento de área de cultivo y cosechado de cultivo respectivamente. El R1 se conforma por la productividad que está en función de la relación entre la cantidad de bioetanol que se produzca y la cantidad hectáreas de caña sembradas. Esta al ser positiva se traduce en un aumento de la demanda de caña. Por su parte el B5 representa el lado de la cadena que muestra la producción de bioetanol de caña, pues su obtención depende la cantidad de caña cosechada que aumenta la cantidad de la fase de alistamiento de caña, a partir de la cual se obtiene el jugo de caña, que es llevado a producción de bioetanol. Esta producción de bioetanol, naturalmente aumenta el inventario o cantidad de bioetanol producido, el cual influye sobre la productividad. EI B3 y B4 representan una acumulación y des-acumulación del bioetanol producido, por la dinámica del mercado que incluye el transporte del producto y su venta o fase final que sería la mezcla con la gasolina para disposición de los consumidores.

Una vez construido el diagrama causal que representa la cadena de suministro a estudiar, se procede entonces a vincular los atributos de los indicadores de sostenibilidad.

A continuación se presenta el diagrama causal (Figura 5) (Anexo), como marco referencial para la evaluación de indicadores de 
sostenibilidad. Este, describe la vinculación de 6 indicadores de sostenibilidad en la cadena de suministro, estos indicadores pertenecientes a los tres pilares de sostenibilidad presentados en la Tabla 2. Estos indicadores son: Consumo de agua, calidad de agua (DBO descargado), número de empleos generados, afectaciones y/o muertes por enfermedades respiratorias, productividad y flexibilidad.

\section{Discusión}

Teniendo en cuenta que el direccionamiento del gobierno nacional es seguir aumentando esta capacidad de producción, es necesario tener en cuenta resaltar que el crecimiento de este tipo de industria en el país ha tenido impactos tanto positivos como negativos en el ámbito económico, ambiental y social dado que estos sistemas de producción son bastante complejos y tienen un gran número de factores que influyen en la sostenibilidad de su producción (Janssen \& Rutz, 2011). Estos impactos generados en la producción de bioetanol, están asociados a distintas etapas de la cadena de suministro de bioetanol y el uso de la comprensión sistémica por medio de diagramas causales permite ver las interrelaciones que tienen las etapas de la cadena de suministro con las posibles afectaciones ambientales y sociales, que bajo una visión lineal no podrían ser observados tan fácilmente.

Colombia actualmente tiene una capacidad instalada de 1.650.000 Litros/día centralizado en los departamentos de Cauca, Valle del Cauca y Risaralda. Sin embargo la producción real se estima en un $85 \%$ de esta capacidad, a esto se le suma el incremento de la demanda de gasolina nacional ya sea por el cierre fronterizo o por incremento del parque automotor, lo cual hace obligatorio para la satisfacción de la demanda interna en algunos casos, la importación de un porcentaje de etanol que permita cubrir esta diferencia de la demanda con la producción. Esto evidencia la necesidad que tiene el país para seguir incrementando la capacidad instalada de producción de bioetanol, este incremento debe realizarse bajo una perspectiva sistémica sostenible.

\section{Conclusiones}

En este trabajo, se planteó una aproximación sistémica de la relación de la producción de bioetanol y su relación con aspectos que miden la sostenibilidad, esto por medio de un diagrama causal que representa la cadena de suministro de bioetanol con indicadores de sostenibilidad establecidos internacionalmente, mostrando así, realimentaciones y relaciones causales de la dinámica del sector y su afectación a la sostenibilidad.

Se percibe que es imperativa la puesta en marcha de un programa de sostenibilidad de producción de biocombustibles en Colombia, ya sea un esquema de certificación o evaluación y seguimiento de los indicadores GBEP. Dado esto se requieren de herramientas que permitan la evaluación de la sostenibilidad es diferentes tiempos y periodos de evaluación, es decir una evaluación prospectiva. Una de estas herramientas es la Metodología de dinámica de sistemas por medio de la cual se pueden capturar hipótesis como la mostrada en este artículo. Sin embargo, se debe ir más allá, se debe identificar los indicadores de sostenibilidad que apliquen para cada contexto y realizar el modelamiento y simulación de distintos escenarios, con el fin de evaluar la sostenibilidad.

Aunque los indicadores proveen información sobre efectos potenciales o reales de las actividades humanas actividades industriales específicas (Dale et al., 2013), en este sentido, para una evaluación exitosa de sostenibilidad con indicadores estos deben haber sido 
escogidos dentro de un contexto bien definido, donde se vincule desde la extracción de las materias primas hasta la distribución o mezcla del biocombustible a evaluar.

\section{Literatura citada}

Asociación de Cultivadores de Caña de Azúcar de Colombia, Asocaña. (2012). Informe anual 2011 - 2012. Cali: Asocaña.

Asociación de Cultivadores de Caña de Azúcar de Colombia, Asocaña. (2016). Informe anual 2015 - 2016. Cali: Asocaña.

Chauvet, M. \& González, R. (2008). Biocombustibles y cultivos biofarmacéuticos: ¿oportunidades o amenazas? Revista El Cotidiano, Redalyc, 23(147), 51-61.

Consorcio CUE, (2012).Capitulo II: Estudio ACV - Impacto Ambiental. Evaluación del ciclo de vida de la cadena de producción de biocombustibles en Colombia. Banco Interamericano de Desarrollo, Ministerio de Minas y Energía, Medellín.

Consejo Nacional de Política Económica y Social, CONPES (2008).Lineamiento de política para promover la producción sostenible de biocombustibles en Colombia. Departamento Nacional de Planeación, Bogotá.

Dale, V.H., Efroymson, R., Kline, K., Langholtz, M., Leiby, P., Oladosu G., Davis, M., Downing, HiIliard, M, (2013). Indicators for assessing socioeconomic sustainability of bioenergy systems: A short list of practical measures. Ecological Indicators 26. 87-102. http://dx.doi.org/10.1016/j. ecolind.2012.10.014

Esteves, B. (2013). Beyond common place biofuels: Social aspects of ethanol, Energy Policy 57(2013)355-362

Espinoza, A., Bautista, S., Narvaez, C., Alfaro, Camargo, M. (2017) Sustainability assessment to support governmental biodiesel policy in Colombia: A system dynamics model. Journal of Cleaner Production 141 (2017) 1145-1163

Flórez, A.M. (2011) Modelo regional de producción y transporte de biocombustibles en Colombia. Tesis Maestría. Universidad Nacional de Colombia Facultad de Minas, Escuela de Sistemas Medellín, Colombia.
Fedesarrollo Colombia. (2012) Garcia, H., Calderon, L. Evaluación de la política de Biocombustibles en Colombia. Fededesarrollo. Recuperado de http://www.fedesarrollo.org.co/wp-content/uploads/2011/08/ Evaluaci\%C3\%B3n-de-la-pol\%C3\%ADtica-deBiocombustibles-en-Colombia.pdf

Federación Nacional de Biocombustibles Fedebiocombustibles (2016). Cifras Informativas del Sector Biocombustibles. Etanol de caña anhidro en 2016.

Forrester J. (1999a). Industrial Dynamics. Waltham: Pegasus Communications, Inc.

Forrester J. (1999b). Urban Dynamics. Waltham: Pegasus Communications, Inc.

Global Bioenergy Partnership, GBEP (2011). The global bioenergy partnership sustainability indicators for bioenergy. Rome: Global Bioenergy Partnership; 2011.

Ibarra, Vega, D.W. \& Redondo, J.M. (2015). Dinámica de Sistemas, una herramienta para la educación ambiental en ingeniería. Revista Luna Azul, 41, 152- 164.

Ibarra, D.W (2017). Modelado para la evaluación de Sostenibilidad en la Cadena de Suministro de Bioetanol. Tesis Doctoral, Ingeniería- Línea Automática. Universidad Nacional de Colombia Sede Manizales Facultad de Ingeniería Eléctrica, Electrónica y Computacional. Manizales, Colombia.

Janssen, R. \& Rutz, D. (2011). Sustainability of biofuels in Latin America: Risks and opportunities. Energy Policy 39, 5717-5725 doi:10.1016/j.enpol.2011.01.047

Sánchez,Ó.J. \& Cardona,C.A.,(2008) .Trends in biotechnological production of fuel ethanol from different feedstocks. Bioresource.Technology.99, 5270-5295.

Scarlat N. \& Dallemand J.F. (2011) Recent developments of biofuels/ bioenergy sustainability certication: a global overview. Energy Policy; 39(3), 1630-46

Sánchez, O. (2008) Síntesis de Esquemas Tecnológicos Integrados Para la Producción Biotecnológica de Alcohol Carburante a Partir de Tres Materias Primas Colombianas. Tesis Doctoral, Departamento de Ingeniería Química Universidad Nacional de Colombia Sede Manizales. 
Valencia, M. \& Cardona, C.A. (2014) The Colombian biofuel supply chains: The assessment of current and promising scenarios based on environmental goals. Energy Policy 67232242
Valencia, M. (2013) Assessment of Greenhouse Gases Emissions Associated To Colombian Biofuels Lifecycle. Tesis Maestría, Departamento de Ingeniería Química Universidad Nacional de Colombia Sede Manizales. 